\title{
The Mechanics of Indentation Induced Lateral Cracking
}

\author{
$\mathrm{Xi} \mathrm{Chen}^{\dagger}$ \\ Department of Civil Engineering and Engineering Mechanics, Columbia University, New York, New York 10027
}

John W. Hutchinson

Division of Engineering and Applied Sciences, Harvard University, Cambridge, Massachusetts 02138

\author{
Anthony G. Evans ${ }^{\star \star}$ \\ Materials Department, University of California, Santa Barbara, California 93106
}

\begin{abstract}
The mechanics governing the lateral cracks that form when a hard object plastically penetrates a ceramic is presented. The roles of indentation load, penetration depth, and work of indentation are all highlighted, as are the influences of the mechanical properties of the material. A closed form solution for cracking induced by expansion of a two-dimensional cavity is used to bring out essential features related to parametric dependence and scaling. The three-dimensional axisymmetric problem for an annular crack driven by a rigid spherical or conical indenter is solved using numerical methods. The region of highest tensile stress is identified corresponding to the location where a crack is most likely to nucleate. This location coincides with the depth below the surface where the crack will expand parallel to the surface under mode I conditions. The solutions have been substantiated by comparison with measurements of the cracks that form upon Vickers indentation. The basic formula for the crack radius has been used to predict trends in cracking upon static penetration and impact by a projectile. In both cases, the extent of the cracking is substantially diminished by increasing the toughness of the material. The yield strength has a much smaller effect. The cracks caused by penetration and the volume removed per impact both decrease marginally at higher yield strength.
\end{abstract}

\section{Introduction}

$\mathrm{W}$ HEN a small indenter plastically penetrates a brittle solid, a pattern of cracks often forms around the impression, as the indenter is removed. Some cracks extend radially outward and penetrate the material normal to the surface. Others nucleate beneath the surface and enlarge laterally, parallel to the surface. ${ }^{1-5}$ Indentation-induced radial cracking has become the basis for a method that can be used to estimate the fracture toughness. ${ }^{2,3,6-10}$ Moreover, detailed analysis has been conducted for the radial cracks that form upon machining and during particle impact, which degrade the strength. ${ }^{11,12}$ Lateral cracks have not been characterized to a comparable extent. Yet, such cracks participate in equally important phenomena. Lateral cracks at the interface between thin films and substrate can be used to estimate the interface toughness. ${ }^{13,14}$ Such cracks are also responsible for the abrasive wear and erosion of ceramics and ceramic coatings. ${ }^{15-18}$ The role of these cracks in the erosion and foreign object damage experienced by thermal barrier ox-

D. Marshall—contributing editor

\footnotetext{
Manuscript No. 10970. Received April 12, 2004; approved November 29, 2004 ${ }^{* *}$ Fellow, American Ceramic Society

Author to whom correspondence should be addressed. e-mail: xichen $@$ civil.columbia.edu
}

ides used in gas turbines, when impacted by small particles in the gas stream, is of current interest. ${ }^{15-18}$ The objective of this article is to present a basic mechanics analysis of lateral cracking (Fig. 1) that can be used to understand and characterize these phenomena: albeit that embellishments will be necessary in some cases, such as in thermal barrier systems ${ }^{16,17}$ that include anisotropy. The focus is on the extension of the cracks (not on initiation). It is implicit that small flaws are always present.

A suite of careful experimental measurements of lateral cracks in transparent ceramics and glasses (symbols in Fig. 2) remains the sole basis for characterization. ${ }^{4}$ Namely, based on these measurements, by using dimensional analysis plus insights from radial cracking, phenomenological expressions relating trends in crack radius with material properties (elastic modulus, the Poisson ratio, toughness, and yield strength) have been deduced. However, the trends in crack size with the individual properties are difficult to de-convolute with adequate fidelity for two reasons. (a) The cracking is affected by non-dimensional groups of properties occurring in unknown combinations with unknown exponents. (b) Only a limited range of measurements are available to calibrate the phenomenological functions. Accordingly, herein, the mechanics of lateral cracks are approached from a fundamental perspective, leading to expressions that explicitly relate crack size to the individual material properties.

Basic insights about lateral cracks are first obtained by deriving analytical results for cylindrical configurations that can be solved in closed form. The analogous problem for the spherical configuration cannot be solved because, while the stress fields surrounding an expanded cavity are known, as well as the mode I stress intensity factor for cracks in the vicinity of the impression, there is no solution for the mode II stress intensity factor. These results for the cylinder give clear indications of trends in material properties. Thereafter, results for indentations are obtained by re-deriving the corresponding non-dimensional groups, and then calculating specific results for the stresses and stress intensity factors by using finite elements. The solutions (lines in Fig. 2) are compared with the experimental measurements.

\section{Cracking Outside a Cylindrical Cavity}

A model two-dimensional problem for cracking due to a cylindrical cavity expanded into an elastic-perfectly plastic solid provides insight into three-dimensional indentation-induced cracks. The advantage of the cavity model is that it can be solved in closed form providing precisely the combination of dimensionless groups that govern the solution and aspects of scaling. This is important since the number of groups that govern the solution is smaller than the total set of dimensionless groups. The analogous three-dimensional model cavity problem would also be relevant, but it cannot be solved in closed form. The assessment is performed by first analyzing the stresses that develop outside 


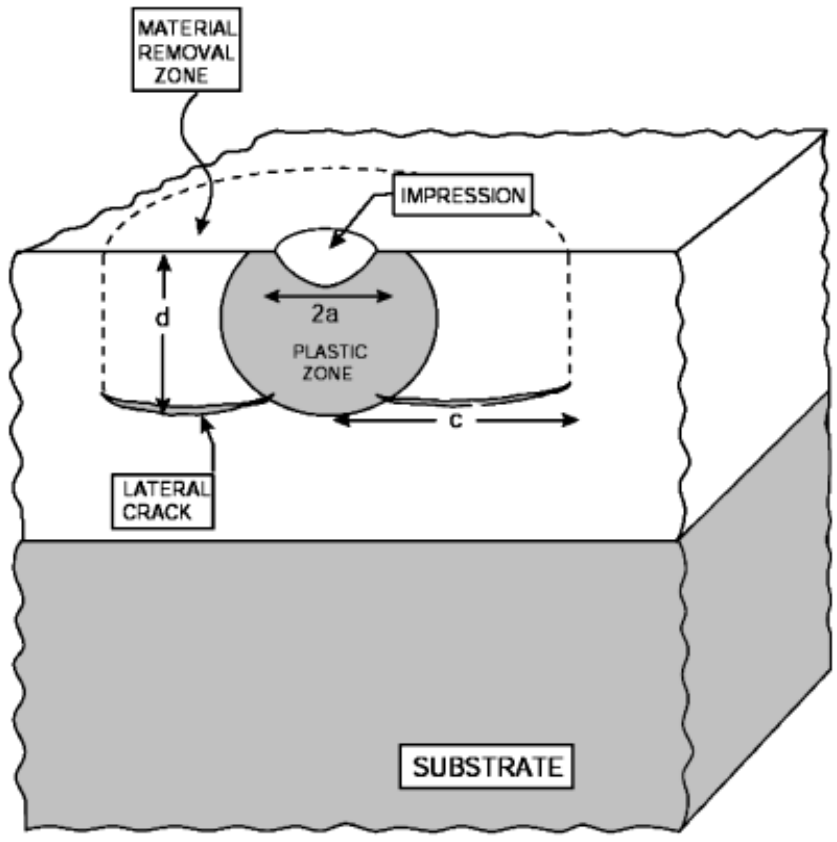

Fig. 1. Schematic showing of the lateral crack system. The spherical indentation (with load $P$ ) on an elastic-plastic solid leaves an impression with projected contact radius $a$. The lateral crack is annular with length (or radius) $c$, which forms just outside the plastic zone, at distance $d$ below the free surface.

the plastic zone upon unloading. Cracks are then introduced and energy release rates and mode mixities ascertained.

To determine the stresses, the cylindrical cavity depicted in Fig. 3 is expanded by internal pressure, $p$, to its current radius $a$, starting from zero. Attention is restricted to elastic-perfectly materials governed by a Mises yield surface (with Young's modulus, $E$, Poisson's ratio, $v$, and yield stress, $\left.\sigma_{Y}\right)$. In the elastically incompressible limit $(v=1 / 2)$, the plane-strain (or cavitation) problem, ${ }^{19,20}$ can be solved in closed form. The solution reveals that the cavity expands at constant (cavitation) pressure: $p^{*}=\lambda \sigma_{\mathrm{Y}}$. The coefficient $\lambda$ is weakly dependent on the yield strain, with $\left(0.001<\sigma_{Y} / E<0.003\right), \lambda \cong 3.70$. The radius of the plastic zone is related to $\lambda$ by

$$
R_{\mathrm{P}} / a=\mathrm{e}^{(\sqrt{3} \lambda-1) / 2} \cong 14.9
$$

The cavity plastic zone size is much larger that the plastic zone surrounding an indent. However, large plastic strains are confined to radii less than about $3 a$. In cylindrical coordinates the inplane stresses outside the plastic zone $\left(r>R_{\mathrm{p}}\right)$ upon unloading are

$$
\sigma_{\theta \theta}=-\sigma_{r r}=\frac{W}{r^{2}} Q
$$

where $W$ is the work per unit length required to create the cavity

$$
W=\pi p^{*} a^{2}=\pi \lambda \sigma_{Y} a^{2}
$$

and

$$
Q=\frac{1}{\pi \lambda}\left(\frac{1}{\sqrt{3}} \mathrm{e}^{\sqrt{3} \lambda-1}-\lambda\right) \cong 10.8
$$

A crack is inserted along $x_{2}=-d$ with its left end at $x_{1}=b$, taken such that the crack lies outside the plastic zone (Fig. 3). The normal and shear stresses acting on the crack plane prior to its formation are readily determined from (2) as

$$
\left(\sigma_{22}, \sigma_{12}\right)=\frac{W Q}{r^{2}}(-\cos 2 \theta, \sin 2 \theta)
$$
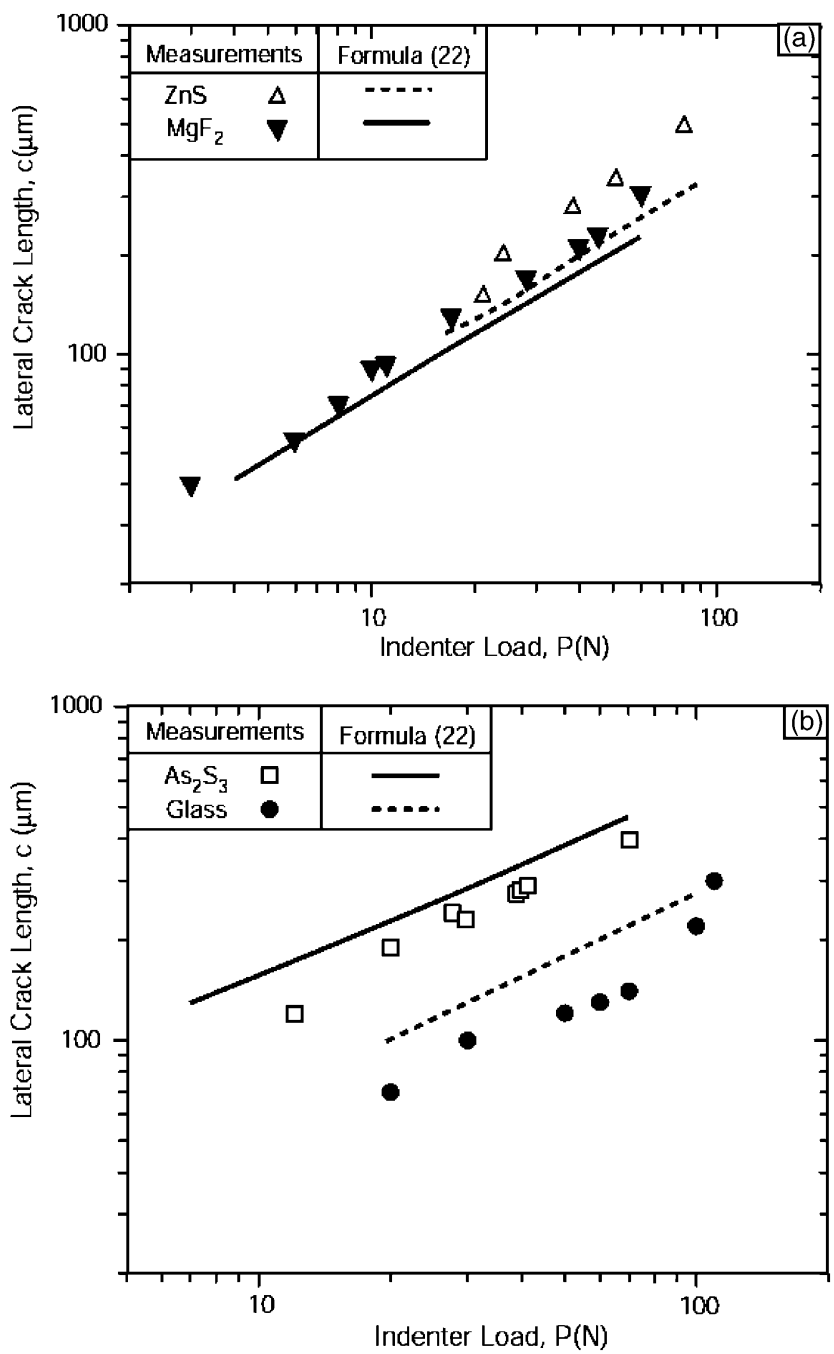

Fig. 2. Plot of lateral crack dimension $(c)$ versus load $(P)$ for four brittle materials: comparison between theory and experiments. The symbols represent experimental data obtained from Marshall et al. ${ }^{4}$ and solid lines are theoretical predication from the present analysis. (a) $\mathrm{MgF}_{2}$ and $\mathrm{ZnS}$ and (b) $\mathrm{As}_{2} \mathrm{~S}_{3}$ and soda lime glass.

where $\theta$ is defined in Fig. 3. The normal stress is positive for $\theta>\pi / 4$. Since the deformations accompanying the formation of the crack are elastic, and ignoring the small interaction of the crack with the cavity itself, the mode I and II stress intensity factors at the right-hand crack tip $(b+c,-d)$ can be written as $^{21}$

$$
\begin{aligned}
\frac{\left(K_{\mathrm{I}}, K_{\mathrm{II}}\right)}{W a^{-3 / 2}} \equiv & \left(q_{\mathrm{I},} q_{\mathrm{II}}\right)=\frac{Q}{\sqrt{\pi c / 2 a}} \\
& \times \int_{-c / 2 a}^{c / 2 a}(-\cos 2 \theta, \sin 2 \theta) \sqrt{\frac{c / 2 a+\xi}{c / 2 a-\xi}} \frac{\mathrm{d} \xi}{(r / a)^{2}}
\end{aligned}
$$

The limits of integration are consistent with $\xi$ as the variable. The corresponding energy release rate is:

$$
\frac{G}{W^{2} /\left(E a^{3}\right)}=\frac{3}{4}\left(q_{\mathrm{I}}^{2}+q_{\mathrm{II}}^{2}\right)
$$

and the associated measure of mode mix $\psi$ is

$$
\tan \psi \equiv \frac{K_{\mathrm{II}}}{K_{\mathrm{I}}}=\frac{q_{\mathrm{II}}}{q_{\mathrm{I}}}
$$




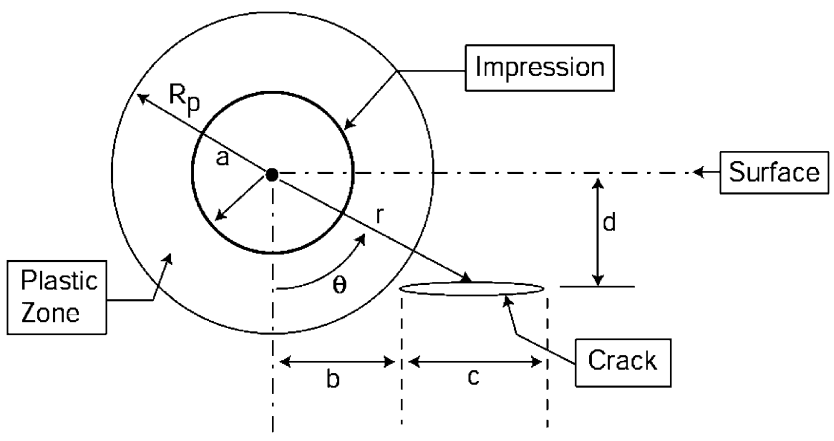

Fig. 3. Schematic drawing of cracking outside a cylindrical cavity.

The normalized energy release rate and the mode mix are plotted as a function of crack length in Fig. 4 for cracks at various depths below the symmetry plane: all having their left end at $b=R_{\mathrm{p}}$. By symmetry, the crack along $x_{2}=0(d=0)$ is necessarily a Mode I crack. At all depths, the energy release rate first increases and then falls as the crack grows, similar to indentation-induced cracks.

The preceding results illuminate indentation-induced crack scaling (although precise details depend on the dimensionality of each problem). For example, to expose the role of the cavity size and the yield stress, by using $W=\pi p^{*} a^{2}$ and $p^{*}=3.70 \sigma_{\mathrm{Y}}$, $G /\left(W^{2} / E a^{3}\right)$, in (7) and Fig. 4 can be re-expressed as:

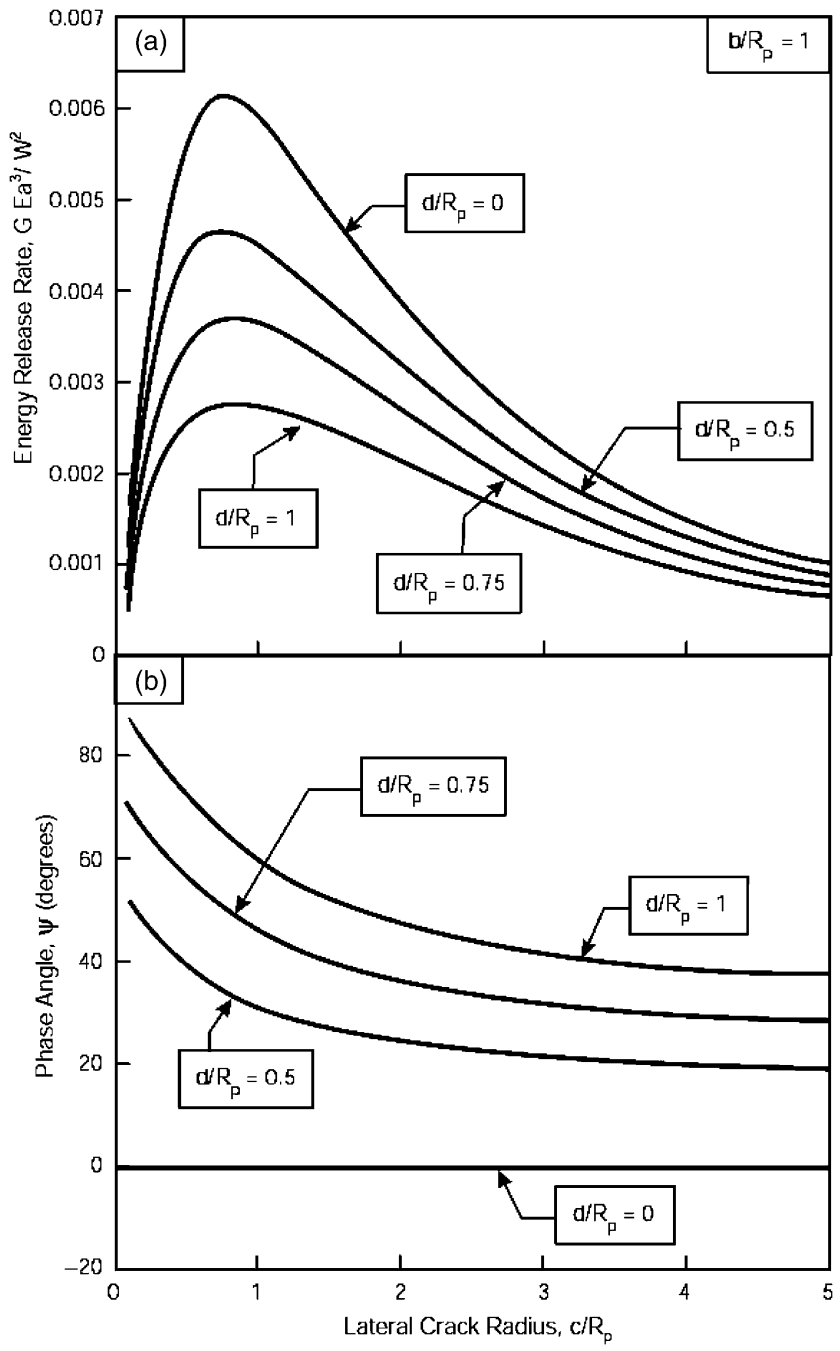

Fig. 4. (a) The dimensionless energy release rate, $G /\left(W^{2} / E a^{3}\right)$, and (b) the mode mix phase angle, $\Psi$, as a function of normalized lateral crack radius, $c / R_{\mathrm{p}}$, for various depths of the crack below the symmetric plane.
$G /\left[\left(3.70 \pi \sigma_{\mathrm{Y}}\right)^{2} a / E\right]$. Alternatively, to express the energy release rate exclusively in terms of the work to create the cavity, relevant to impact indentation of a substrate, then $G /\left(W^{2} / E a^{3}\right)$ becomes $G /\left[W^{1 / 2}\left(3.70 \pi \sigma_{Y}\right)^{3 / 2} / E\right]$. Counterparts to these normalizations will be presented below for indentation-induced cracks.

\section{Basic Relations for Indentation of a Half-Space}

When the penetration of a spherical or conical indenter into an elastic/perfectly-plastic half-space is plasticity dominated, wellknown results ${ }^{22}$ relate the indentation load, $P$, contact radius, $a$, and indentation depth, $\delta$ :

$$
P=k \pi a^{2} \sigma_{\mathrm{Y}}
$$

For shallow indents created by a spherical indenter of radius $R$ :

$$
\delta=a^{2} / 2 R
$$

while for conical indenters with cone angle $\pi / 2-\beta$ :

$$
\delta=a \tan \beta
$$

In order to obtain representative results and make connections between spherical and conical indentations, the effect of plastic pile-up is ignored. The effect of pile-up (or elastic sink-in) and work hardening are discussed in Section V. The hardness of the material is

$$
H \equiv P /\left(\pi a^{2}\right)=k \sigma_{Y}
$$

For conical indenters, the coefficient $k$ depends weakly on the yield strain and the cone angle: for many applications it is sufficient to use, $k=3$. This choice also applies for spherical indenters (with minor departures dependent on the yield strain and on $a / R)$. Procedures to account for the effects of yield strain and strain hardening will be discussed in the next section.

The work to create an indent, volume $V$, is

$$
W=\int P \mathrm{~d} \delta=k \sigma_{\mathrm{Y}} \int \pi a^{2} \mathrm{~d} \delta=k \sigma_{\mathrm{Y}} V
$$

For future scaling purposes, note that the work can be expressed in terms of $\delta, P$, and $a$, independently. For spherical indentation:

$$
W=\pi k \sigma_{\mathrm{Y}} R \delta^{2}=\frac{P^{2}}{4 \pi R k \sigma_{\mathrm{Y}}}=\frac{\pi}{4} k \sigma_{\mathrm{Y}} \frac{a^{4}}{R}
$$

For conical indentations:

$$
W=\frac{\pi}{3} \frac{k \sigma_{Y} \delta^{3}}{\tan ^{2} \beta}=\frac{\tan \beta}{3 \sqrt{\pi}} \frac{P^{3 / 2}}{\sqrt{k \sigma_{Y}}}=\frac{\pi}{3} \tan \beta k \sigma_{Y} a^{3}
$$

Neglecting the weak dependence of $k$ on indenter geometry and yield strain, Eqs. (8) and (13) imply an equivalence between conical and spherical indentation: namely, indents with identical contact radius $a$ and volume $V$ require the same load $P$ and work $W$ and have the same hardness $H$. The corresponding stress fields outside the plastic zone created by the indent are also essentially the same. The relation between the cone angle $\beta$ and $a / R$ for the spherical indenter, such that the two indentations have identical $a$ and $V$, is plotted in Fig. 5. The conical equivalent of the Berkovich indenter corresponds to $a / R=0.44$, while that for the Vickers indenter corresponds to $a / R=0.49$.

\section{Indentation-Induced Cracks in Elastic-Perfectly Plastic Materials}

Lateral cracking from indentations can be analyzed in two parts. (i) Determination of the stresses induced by indentation of an 


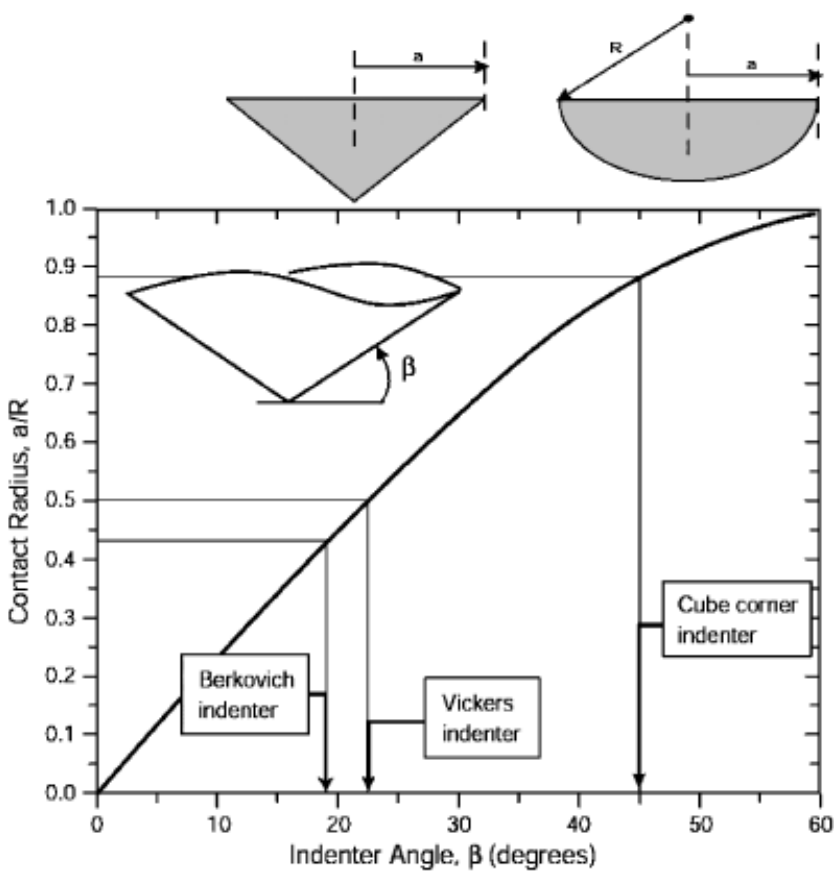

Fig. 5. The relationship governing conical (cone angle $\beta$ ) and spherical indentation (with contact radius/indenter radius ratio $a / R$ ), such that the two indentations create the same contact radius and volume.

elastic-perfectly plastic half-space after unloading (with no crack present). (ii) Calculation of the stress intensity factors for an annular crack in the stress field created by the indent. The cracks are assumed to form outside the plastic zone, where the residual stresses acting on the crack plane are tensile. Both parts of the problem are analyzed numerically, by using the finite element code ABAQUS Standard, ${ }^{23}$ and carried out within a finite strain framework, accounting for finite geometry changes. The energy release rate is calculated from the J-Integral around the crack front.

The distribution of the residual stress component $\sigma_{22}$ surrounding a spherical indentation, following load removal, is illustrated in Fig. 6. The plastic zone extends out to about twice the contact radius. The maximum tensile $\sigma_{22}$ occurs near the boundary of this zone at a depth roughly equal to the contact radius. Cracks destined to propagate parallel to the interface are most likely to be nucleated in this relatively small region. It will emerge that the depth of an annular crack parallel to the surface corresponding to mode I conditions is also at this depth.

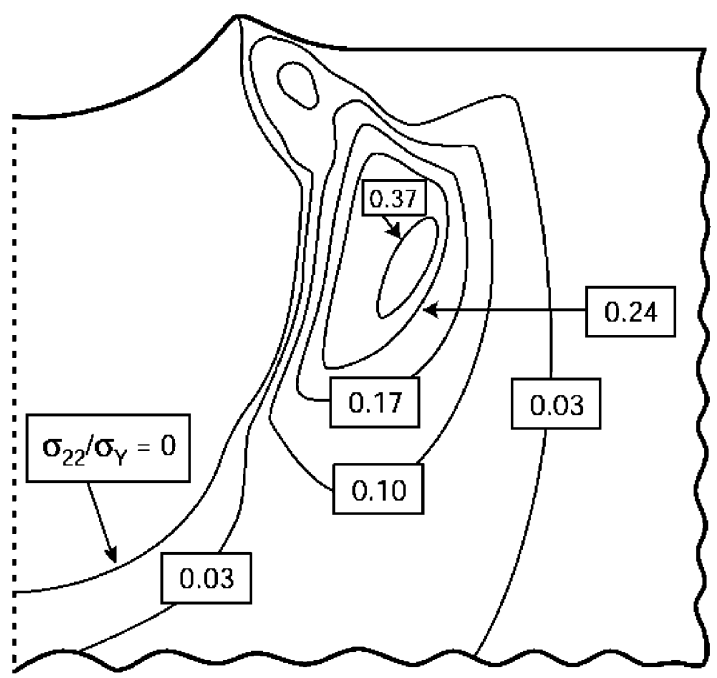

Fig. 6. Contour plot of the residual stress component normal to the lateral crack system $\left(\sigma_{22}\right)$ caused by spherical indentation, with $a$ / $R=0.9$.
The normalization of the energy release rate at the outer tip of the annular crack surrounding the spherical indent in Fig. 1 is:

$$
\frac{G}{W^{2} /\left(E a^{5}\right)}=f\left(\frac{c}{a}, \frac{a}{R}, \frac{d}{a}, \frac{b}{a}\right)
$$

This scaling is analogous to that for the model cylindrical voidinduced crack except that here $W$ is the total work to create the indentation, not the work per unit length. One important implication of (16), affecting trends in crack size with material properties, is that $f$ is not explicitly dependent on the yield strength (it enters implicitly through its influence on $W$, as well as on $a$, if $P$ is prescribed). Moreover, direct numerical calculation has verified that there is no dependence of $f$ on $\sigma_{Y} / E$. The normalization for the conical indenter has the same form as (16) with the dependence on $a / R$ replaced by a dependence on $\beta$.

Results for the normalized energy release rate as a function of crack length, computed from the finite element analysis, are presented in Fig. 7 for four values of $a / R$ (all with $d / a \approx 1$ and $b /$ $a \cong 1.5$ ). The left tip of each crack lies within the zone of high tensile stress noted in conjunction with Fig. 6. The normalized energy release rate and the mode mix for cracks at various depths below the free surface are plotted on Fig. 8 (in each case for $a / R=0.7$ and $b / a=1.5$ ). Note that the crack depth associated with both the largest energy release rate and with mode I conditions is $d / a \cong 1$; these two features hold for a wide range of $a / R$. Thus, cracks at this depth are favored not only because it coincides with the largest tensile stress but also because, once nucleated, cracks must propagate in mode I (parallel to the surface). Subsequently, we focus on spherical indentation with $a /$ $R=0.5$ since this choice corresponds closely to either Berkovich or Vickers indenters (cf. Fig. 5). Comparisons to be made later with experimental data will be based on Vickers indentations.

The normalized energy release rate as a function of crack length for $a / R=0.5, d / a=1$, and $b / a=1.5$ is plotted on Fig. 9 . The peak energy release rate at $c / a \cong 0.6$ is

$$
\frac{G_{\max }}{W^{2} /\left(E a^{5}\right)} \cong 0.11
$$

The following function has been fit to the results in Fig. 9 to the right of the peak (in the range $0.6 \leq c / a \leq 4$ ):

$$
\frac{G}{W^{2} /\left(E a^{5}\right)}=0.10 \mathrm{e}^{-((c / a-0.75) / 0.65)}
$$

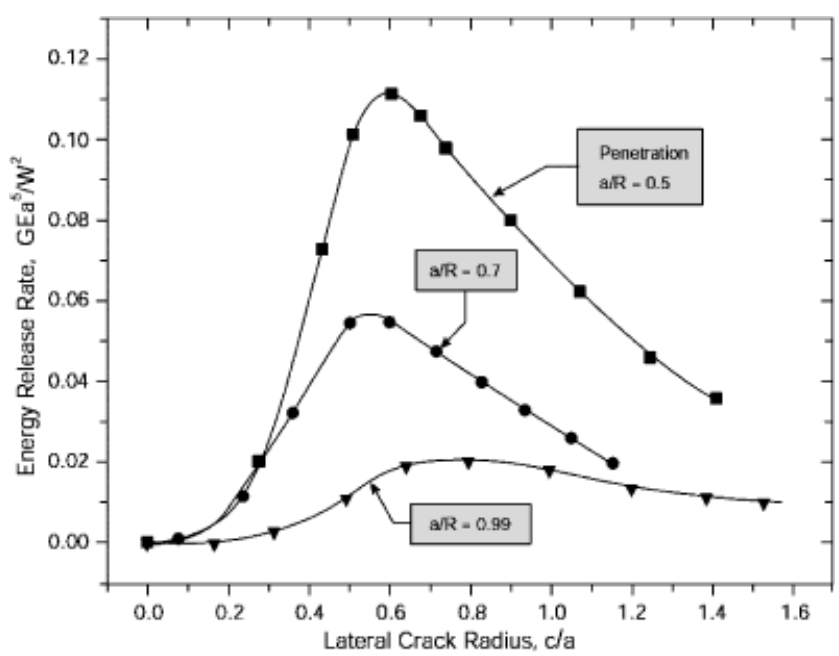

Fig. 7. Normalized energy release rate $G /\left(W^{2} / E a^{5}\right)$ as a function of lateral crack length $c / a$ for three values of contact radius $a / R$ caused by spherical indentation. The cracks are aligned with the preferred cracking path, where $G$ attains maximum and $K_{\mathrm{II}}=0$ (for given $a / R$ ), all with $d /$ $a \approx 1$ and $b / a \cong 1.5$ (see Fig. 8). 

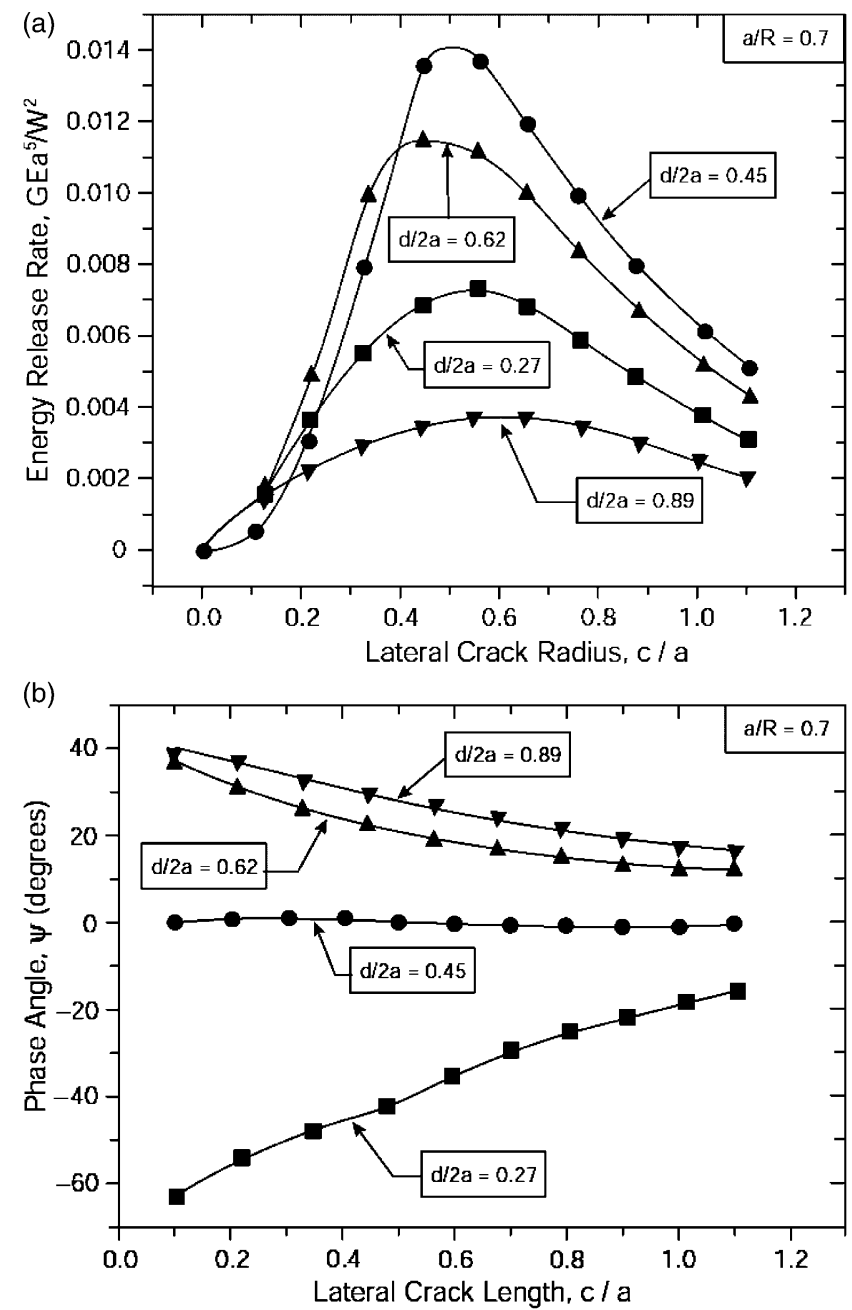

Fig. 8. (a) The dimensionless energy release rate, $G /\left(W^{2} / E a^{5}\right)$, and (b) the mode mix phase angle, $\psi$, as a function of normalized lateral crack length, $c / a$, for various depths of the crack below the free surface (for $a /$ $R=0.7$ and $b / a=1.5$ ).

The accuracy of this fitting function is evident in the figure. However, it is not useful for assessing trends in cracking with material properties, discussed in the next section. Instead, a power law relationship is required. The best fit to a power law is given by:

$$
\frac{G}{W^{2} /\left(E a^{5}\right)} \approx 0.1(a / c)^{4}
$$

as also plotted on Fig. 9. The fit is good but imperfect. Over the relevant range of crack size $(c / a \leq 4)$, it is deemed sufficiently accurate to capture the material property trends described below.

If $\Gamma_{I c}$ is the mode I toughness of the material, a necessary condition for the propagation of a crack is $G_{\max }>\Gamma_{\mathrm{I} c}$. Consequently, from (17), the work of penetration at the cracking threshold is,

$$
W_{\mathrm{th}}^{2} /\left(E a^{5}\right)=9.1 \Gamma_{\mathrm{I} c}
$$

Assuming that the work exceeds the threshold, the crack propagates outwards and then arrests at the crack length $c$ corresponding to $G=\Gamma_{\mathrm{I} c}$, which by $(18 a)$ is

$$
\frac{c}{a}=0.75-0.65 \ln \left(\frac{10 \Gamma_{\mathrm{I} c}}{W^{2} /\left(E a^{5}\right)}\right)
$$

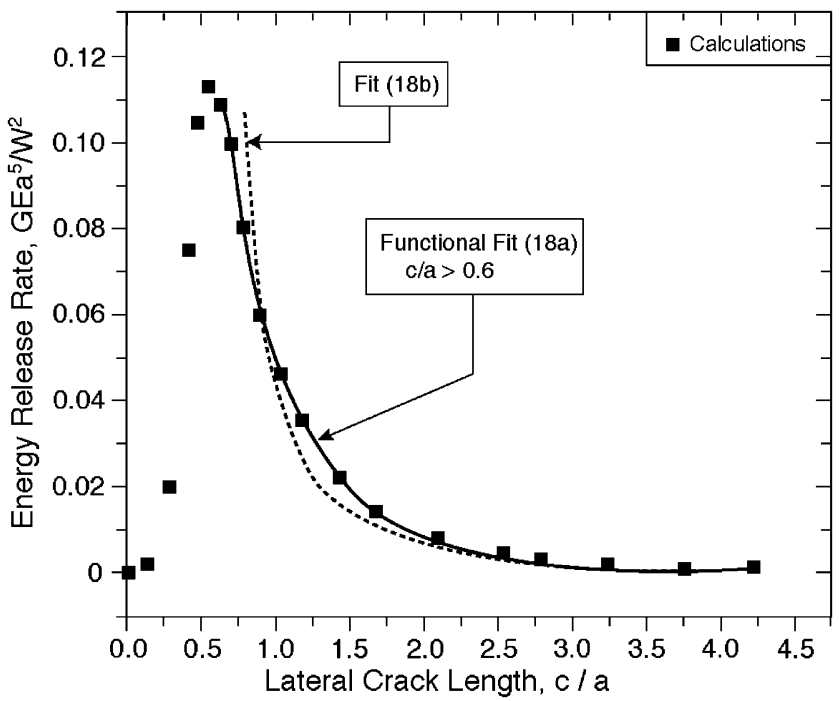

Fig. 9. The normalized energy release rate $G /\left(W^{2} / E a^{5}\right)$ as a function of lateral crack length $c / a$ for Vickers indenter $(a / R=0.5, d / a=1$, and $b /$ $a=1.5$ ). Symbols represent results obtained from the finite element analysis, and solid line is the functional fit from Eq. (18a).

or by $(18 b)$ is

$$
c \approx \frac{0.22 W}{\sqrt{E \Gamma_{\mathrm{I} c}} a^{3 / 2}}
$$

In summary, for either a Berkovich or a Vickers indentation (or a spherical indentation with $a / R \approx 0.5$ ), the potential crack plane which simultaneously has the largest energy release rate and mode I conditions is located below the surface at depth, $d / a \cong 1$. There is a maximum energy release given by (17). If the toughness exceeds this maximum, a crack will not form. If it is lower, a crack may nucleate. If it does, it would propagate and arrest at an outer radius $b+c$, where $b / a \cong 1.5$ and $c / a$ is given by (20).

\section{Application and Comparison with Measurements}

The non-dimensional parameter characterizing the indentation, $W^{2} /\left(E a^{5}\right)$, can be expressed in the following alternative ways that highlight the roles of the load, $P$, the indent radius, $a$, and the work of indentation, $W$, respectively:

$$
\begin{aligned}
W^{2} /\left(E a^{5}\right) & =\frac{\tan ^{2} \beta}{9} \frac{\left(\pi k \sigma_{\mathrm{Y}}\right)^{3 / 2} P^{1 / 2}}{E} \\
& =\frac{\tan ^{2} \beta}{9} \frac{\left(\pi k \sigma_{\mathrm{Y}}\right)^{2} a}{E} \\
& =\frac{3^{1 / 3} \tan ^{5 / 3} \beta}{9} \frac{\left(\pi k \sigma_{\mathrm{Y}}\right)^{5 / 3} W^{1 / 3}}{E}
\end{aligned}
$$

By using (21) to convert the lateral crack size solution in (20b) to a trend in crack size with Vickers indentation load, the trend in the crack size with material properties, at fixed load, can be ascertained as:

$$
c \approx\left\{\left(\frac{0.1}{9} \frac{\tan ^{2} \beta}{\sqrt{\pi k}}\right)\left[\frac{P^{2}}{E \Gamma_{\mathrm{I} c}} \sqrt{\frac{P}{\sigma_{\mathrm{Y}}}}\right]\right\}^{1 / 4}
$$

Note that the crack size increases as the toughness decreases and as the yield strength increases. Incorporating the data for toughness and hardness from Marshall et al., ${ }^{4}$ the crack lengths predicted by the present model can be superposed on Fig. 2. Note that, in all cases, the measured trends in crack length are consistent with formula (22). That is, the formula predicts that the cracks in $\mathrm{ZnS}$ should be slightly larger than those in $\mathrm{MgF}_{2}$ (Fig. 2(a)), and the cracks in $\mathrm{As}_{2} \mathrm{~S}_{3}$ be appreciably larger than 


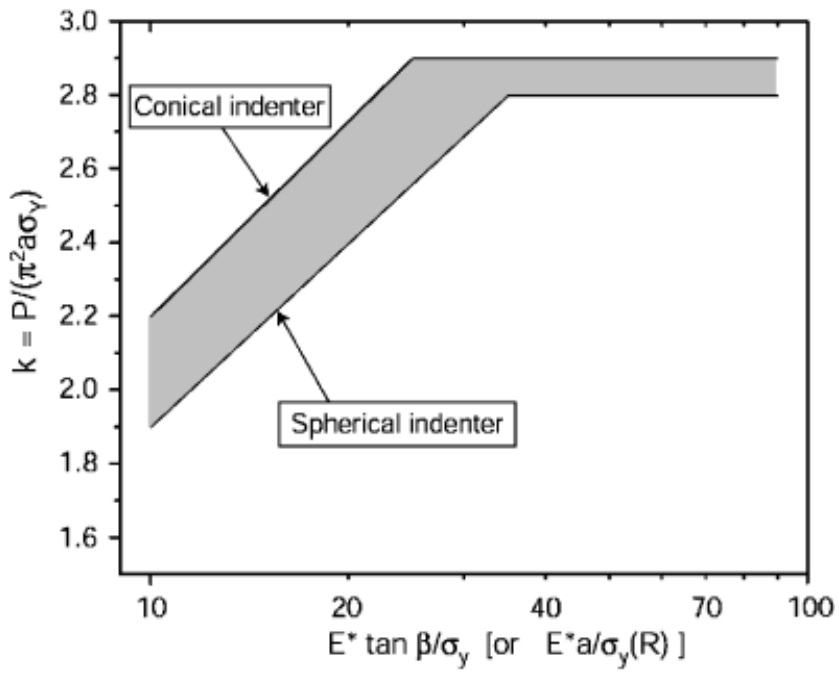

Fig. 10. The spread of experimental data in $k$ (shown in the shaded region) measured from conical and spherical indentations, which are summarized by the classic textbook of Johnson. ${ }^{22}$ The dimensionless indentation strain is $E^{*} \tan \beta / \sigma_{\mathrm{Y}}$ for conical indenter and $E^{*} a /\left(\sigma_{\mathrm{Y}} R\right)$ for spherical indenter, respectively.

those in glass (Fig. 2(b)). Both predictions are in accordance with the measurements. Given the uncertainty in the yield strength (hardness) and toughness for these materials, the correspondence is considered adequate for further application of the model.

The cracking that occurs upon projectile impact can be predicted by relating the plastic work of penetration, $W$, to the kinetic energy of the projectile: $W \approx 0.8 m v^{2} / 2$, (with $m$ the projectile mass and $v$ its velocity): neglecting strain rate hardening,

$$
c \approx 0.35\left\{\pi^{2} k^{2} \tan ^{2} \beta\left[\frac{\left(m v^{2}\right)^{10} \sigma_{\mathrm{Y}}^{2}}{\left(E \Gamma_{\mathrm{I} c}\right)^{6}}\right]\right\}^{1 / 24}
$$

Since the material between the crack and the surface is susceptible to removal (Fig. 1), the volume of material removed per impact, $V_{\text {impact, }}$ scales as:

$$
\begin{aligned}
V_{\text {impact }} & =\pi c^{2} a \\
& \approx 0.41\left\{\frac{1}{\pi^{2} k^{2} \tan ^{2} \beta}\left[\frac{\left(m v^{2}\right)^{14}}{\left(E \Gamma_{\mathrm{I} c}\right)^{6} \sigma_{\mathrm{Y}}^{2}}\right]\right\}^{1 / 12}
\end{aligned}
$$

Consequently, to minimize the erosion rate, the material should have high toughness. The influence of the yield strength is negligible: countering the commonly held belief that the erosion resistance is improved by choosing materials with high hardness. Anisotropy of the type found in thermal barrier oxides will affect the specifics but not the trends with material properties. The implications are discussed elsewhere. ${ }^{16,17}$

The foregoing results can be elaborated by using Johnson's ${ }^{22}$ (p. 167) solutions to account for the dependence of $k$ on $\sigma_{\mathrm{Y}} / E$, indenter elasticity, cone angle, or the depth of a spherical indentation. From several sources, Fig. 10 reproduces the spread in $k$ as summarized by Johnson. Here $E^{*}$ is a modulus dependent on both substrate $(E, v)$ and indenter $\left(E_{\mathrm{i}}, v_{\mathrm{i}}\right)$ elastic properties: $1 / E^{*} \equiv\left(1-v^{2}\right) / E+\left(1-v_{\mathrm{i}}^{2}\right) / E_{\mathrm{i}}$. Note that $E^{*}$ reduces to the plane strain modulus of the substrate when the indenter is rigid (as in the present study). Johnson also discusses Tabor's rules $^{25}$ for assigning a value to $\sigma_{Y}$ for strain hardening materi-

During impact, although a majority of the kinetic energy is converted to the plastic work of penetration, a certain amount is lost in forms of stress wave and rebound energy. als. For example, for a cone with $\beta=19.7^{\circ}$, representative of a Berkovich indenter, Tabor recommends that $\sigma_{Y}$ is identified with the stress at a compressive strain of $8 \%$. For a spherical indenter, $\sigma_{\mathrm{Y}}$ is taken as the stress at a compressive strain $0.2 a / R$.

\section{Conclusion}

A combined analytical-numerical analysis has been presented of the lateral cracks that form when a hard object plastically penetrates a ceramic. The radii of cracks predicted to form upon Vickers indentation have been compared with experimental measurements. An adequate correspondence between the measurements and predictions provides a rationale for using the model to predict trends in cracking for various scenarios. The basic formulas relating the crack radius to the load and work of penetration have been used to predict trends with material properties for two situations: (a) static penetration up to a specified load and (b) impact by a projectile. In both cases, cracking is diminished by increasing the toughness of the ceramic (consistent with many previous findings). The yield strength has essentially no effect.

\section{References}

${ }^{1}$ R. F. Cook and G. M. Pharr, "Direct Observation and Analysis of Indentation Cracking in Glasses and Ceramics," J. Am. Ceram. Soc., 73, 787-817 (1990).

${ }^{2}$ G. R. Anstis, P. Chantikul, B. R. Lawn, and D. B. Marshall, "A Critical Evaluation of Indentation Techniques for Measuring Fracture Toughness: I, Direct Crack Measurements," J. Am. Ceram. Soc, 64, 533-45 (1981).

${ }^{3}$ B. R. Lawn, A. G. Evans, and D. B. Marshall, "Elastic/Plastic Indentation Damage in Ceramics: The Median/Radial Crack System," J. Am. Ceram. Soc, 63, 574-80 (1980).

${ }^{4}$ D. B. Marshall, B. R. Lawn, and A. G. Evans, "Elastic/Plastic Indentation Damage in Ceramics: The Lateral Crack System," J. Am. Ceram. Soc, 65, 561-6 (1982).

${ }^{5}$ B. R. Lawn and E. R. Fuller, "Equilibrium Penny-Like Cracks in Indentation Fracture," J. Mater. Sci., 10, 2016-20 (1975).

${ }^{6}$ B. R. Lawn and A. G. Evans, "A Model for Crack Initiation in Elastic/Plastic Indentation Fields," J. Mater. Sci., 12, 2195-201 (1977).

${ }^{7}$ D. B. Marshall, "Controlled Surface Flaws in Ceramics: A Comparison of Knoop and Vickers Indentation," J. Am. Ceram. Soc., 66, 127-33 (1983).

${ }^{8}$ A. G. Evans and E. A. Charles, "Fracture Toughness Determination by Indentation," J. Am. Ceram. Soc., 59, 371-6 (1976).

${ }^{9}$ K. Niihara, "A Fracture Mechanics Analysis of Indentation-Induced Palmqvist Cracks in Ceramics," J. Mater. Sci. Lett., 2, 221-3 (1983).

${ }^{10} \mathrm{~J}$. Lankford, "Indentation Microfracture in the Palmqvist Crack Regime: Implications for Fracture Toughness Evaluation by the Indentation Method," J. Mater. Sci. Lett., 1, 493-5 (1982).

${ }^{11}$ A. G. Evans and T. R. Wilshaw, "Quasi-Static Solid-Particle Damage in Brittle Solids," Acta Mater., 24, 939-45 (1976).

${ }^{12}$ B. R. Lawn, "Indentation of Ceramics with Spheres: A Century of Hertz," J. Am. Ceram. Soc., 81, 1977-85 (1998).

${ }^{13}$ A. Vasinonta and J. L. Beuth, "Measurement of Interfacial Toughness in Thermal Barrier Coating Systems by Indentation," Eng. Fract. Mech., 68, 843-60 (2001).

${ }^{14}$ M. D. Drory and J. W. Hutchinson, "Measurement of the Adhesion of a Brittle Film on a Ductile Substrate by Indentation," Proc. R. Soc. London, A452, 2319-41 (1996).

${ }^{15}$ A. G. Evans, D. R. Mumm, J. W. Hutchinson, G. H. Meier, and F. S. Petit, "Mechanisms Controlling the Durability of Thermal Barrier Coatings," Progr. Mater. Sci., 46, 505-53 (2001).

${ }^{16}$ X. Chen, R. Wang, N. Yao, A. G. Evans, J. W. Hutchinson, and R. W. Bruce, "Foreign Object Damage in a Thermal Barrier System: Mechanisms and Simulations," Mater. Sci. Eng. A, 352, 221-31 (2003).

${ }^{17}$ X. Chen, M. Y. He, I. Spitsberg, N. A. Fleck, J. W. Hutchinson, and A. G Evans, "Mechanisms Governing the High Temperature Erosion of Thermal Barrier Coatings Used in Gas Turbines," Wear, 256, 735-46 (2004).

${ }^{18}$ X. Chen, J. W. Hutchinson, and A. G. Evans, "Simulation of the High Temperature Impression of Thermal Barrier Coatings with Columnar Microstructure," Acta Mater., 52, 565-71 (2004).

${ }^{19}$ Y. Huang, J. W. Hutchinson, and V. Tvergaard, "Cavitation Instabilities in Elastic-Plastic Solids," J. Mech. Phys. Solids, 39, 223-41 (1991).

${ }^{20} \mathrm{R}$. Hill, The Mathematical Theory of Plasticity. Clarendon Press, Oxford, 1950.

${ }^{21}$ H. Tada, P. C. Paris, and G. R. Irwin, The Stress Analysis of Cracks Handbook. ASME Press, New York, 2001.

${ }^{22}$ K. L. Johnson, Contact Mechanics. Cambridge University Press, Cambridge, 1985.

${ }^{23}$ ABAQUS, ABAQUS 5.8 User's Manual. ABAQUS Inc., Pawtucket, RI, 1998

${ }^{24} \mathrm{X}$. Chen and J. W. Hutchinson, "Particle Impact on Metal Substrates with Application to Foreign Object Damage to Aircraft Engines," J. Mech. Phys. Solids, 50, 2669-90 (2002).

${ }^{25}$ D. Tabor, Hardness of Metals. Oxford University Press, Oxford, 1951 